\title{
Un paso más allá en la planificación docente uni- versitaria: el plan de acción tutorial
}

\author{
Gema Alcolea DíAZ \\ gema.alcolea@ajz.ucm.es \\ CES Felipe II, Universidad Complutense de Madrid \\ María José PÉREz SERRANO \\ mariajoseperezserrano@pdi.ucm.es \\ Universidad Complutense de Madrid
}

Recibido: 10/07/2012

Aceptado: 09/10/2012

\section{Resumen}

El EEES ha pregonado un cambio global de paradigma universitario basado en el aprendizaje y en el trabajo autónomo del discente, situando entre sus elementos centrales la acción tutorial. El presente trabajo se basa en el estudio realizado con un grupo de alumnos con el que se han analizado necesidades y aspiraciones de cara a las tutorías, su uso, resultados y la visión de la actual acción tutorial. Se añade la mirada de un grupo de profesores sobre su labor en este campo. El profesor precisa guías de orientación y herramientas para realizar su nueva función. El objetivo es encontrar una salida práctica, una guía de trabajo a una acción tutorial regenerada, revalorizada, desempolvada y sacada del baúl de la burocracia y que es mirada con deseo de verdadera utilidad.

Palabras clave: EEES, acción tutorial, innovación docente, Plan de acción tutorial, Universidad.

\section{A Step Further in University Teaching Planning: the Tutorial Action Plan}

\begin{abstract}
The EHEA has touted a global change of university paradigm based on learning and self-academic work of the student, establishing the Tutorial action plan among its central elements. This article is based on the study made with a students' group, that it has used for analyze needs and aspirations about the tutorships: just like to know uses, results and the vision of the current tutorial process. We add the opinion of a teachers' group on their work in this field. The teacher needs accurate guidance and tools to perform their new role. The goal is to find a practical, a working guide to action tutorial regenerated, reinvigorated, dusted off, and taken out of old bureaucracy and that is regarded with desire of genuine usefulness.
\end{abstract}

Keywords: EHEA, tutorial action, teaching innovation, Tutorial Action Plan, University.

\section{Referencia normalizada}

ALCOLEA DÍAZ, Gema y PÉREZ SERRANO, María José (2012): “Un paso más allá en la planificación docente universitaria: el plan de acción tutorial". Estudios sobre el mensaje periodístico. Vol. 18, núm. especial octubre, págs.: 45-55. Madrid, Servicio de Publicaciones de la Universidad Complutense.

Sumario: 1. Introducción. 2. Metodología. 3. Visión del alumnado y del profesorado del estado de la cuestión; 3.1. Valoración por el alumnado; 3.2. Punto de vista del profesorado. 4. Plan de Acción Tutorial: un sistema dinámico; 4.1. Planteamiento preliminar; 4.2. Fases y cronología del PAT. 5. Conclusiones. 6. Referencias bibliográficas.

\section{Introducción}

Todos los docentes se han visto implicados en la implantación del Espacio Europeo de Educación Superior (EEES), del que se espera una modificación sustancial del rol de estudiantes y profesores. Un nuevo marco universitario que, entre otros pivotes del 
modelo docente basado en el aprendizaje y en el trabajo autónomo del discente, ha situado en el centro del vendaval renovador la acción tutorial.

Una acción que debía superar su carácter burocrático, revalorizando su función dentro de la secuencia docente y colocándose en el nivel de importancia que le corresponde como elemento fundamental para una educación de calidad (Alcolea y Pérez Serrano, 2010).

Algunas universidades cuentan con planes integrales de Sistemas de Tutoría pero, lejos de esas realidades, son muchos los profesores universitarios que se preguntan qué es lo que se espera de ellos una vez que están inmersos en este nuevo modelo, cómo afrontan estas tutorías que buscan un corte diferente y con las que se pretende promover el autoaprendizaje.

Masificación universitaria, premura de tiempos y espacios, sentimiento de anonimato y de imposibilidad de conseguir personalización educativa, "motiva la falta de iniciativas a este respecto [...] pero son precisamente de estos hechos o situaciones mencionadas, de los que se deriva la necesidad de la tutoría como un medio de individualización y calidad educativa que ayude a contrarrestar estos adversos factores" (García Ramos y Gálvez Hernández, 1996). Las tutorías universitarias se han reducido en muchos casos a un proceso que es resultado de la imposición de la dirección académica y que, en muchas ocasiones, es escasamente valorado por los órganos competentes.

El profesor necesita herramientas que le permitan realizar su nueva función, puesto que "para cambiar el paradigma de la enseñanza universitaria no basta con la voluntad, el voluntarismo a veces, de los profesores que, aunque puede impulsar los cambios, es insuficiente" (Rodríguez Uría, 2007). El objetivo es lograr una guía de trabajo para la nueva acción tutorial, labor absolutamente necesaria para que el EEES no caiga en una de esas conocidas batallas que se libran para que todo siga como está.

\section{Metodología}

Este artículo ofrece el análisis del estado de la cuestión y la plasmación en un sistema integral de tutorías para el Grado de Comunicación Audiovisual del CES Felipe II, centro adscrito a la Universidad Complutense de Madrid. El objetivo último es que este sistema no se quede en un mero desiderátum, sino que alcance un grado tal de utilidad que suponga que los beneficios que conlleva este tipo de mejora de la calidad docente, desde el aumento del rendimiento educativo o el desarrollo personal y social del alumnado, hasta cuestiones como la imagen de calidad que la Universidad proyecta al exterior, se hagan realmente patentes.

El trabajo surge de un Proyecto de Innovación y Mejora de la Calidad Docente y el hecho de acotar dicha propuesta al Grado en Comunicación Audiovisual se ha debido a que tiene que producir una completa adaptación de los planes de acción tutorial a las necesidades y peculiaridades de cada carrera, más allá de la lógica existencia de unos objetivos comunes generales.

La finalidad principal ha sido la consecución de una Propuesta para la implantación de un Sistema de Tutoría en Comunicación Audiovisual, finalmente concretado en un Plan de Acción Tutorial (PAT) para el citado Centro, plasmada en un doble objetivo: 
- Conocimiento del estado de la cuestión:

- estudio y valoración de la acción tutorial,

- comprobación del estado y situación de las tutorías,

- análisis de las necesidades del alumnado,

- identificación de otras necesidades para el diseño del PAT.

- Diseño del PAT.

No obstante, podemos decir que la finalidad última de este trabajo ha sido doble, puesto que por un lado se perseguían los objetivos propios del proyecto pero, por otro lado, se suma la consecución de los objetivos de un Sistema de Tutoría (Díaz-Guilera, 2004):

- mejora de la calidad de la titulación,

- mejora del entorno de aprendizaje,

- calidad de la oferta extracurricular,

- satisfacción de los estudiantes,

- rendimiento educativo,

- mejora de las relaciones alumnos-profesores,

- inserción laboral,

- desarrollo personal y social,

- unificación y coordinación del proceso tutorial.

Si además tenemos en cuenta que la función tutorial en el contexto de la enseñanza superior es considerada en los últimos tiempos una de las estrategias de mejora más importantes y que las diferentes acciones que se han desarrollado en este ámbito han estado asociadas a la gestión de la calidad de las universidades, con la implantación del Sistema de Tutoría se logra una mejora de la imagen pública, permitiendo una proyección externa de calidad de la Universidad y la fidelización de los estudiantes. Actualmente, "es difícil hablar de una universidad de mayor calidad sin hacer referencia a una serie de servicios de apoyo al estudiante que le faciliten el proceso formativo" (Miraflores Gómez, 2004).

El conocimiento previo del estado de la cuestión y la identificación de necesidades es una metodología que se sustenta en los teóricos del Iluminismo (Modelo Iluminativo, denominado submodelo del modelo de evaluación basado en la negociación), quienes argumentan que "para diseñar e implementar un modelo de trabajo en un programa, institución o curso específico, se requiere, como primer paso, conocer el proceso de lo existente y posteriormente, realizar un proceso de identificación de necesidades" (Martínez Rodríguez, 2011).

Con el objetivo de conocer la experiencia de la acción tutorial, de detectar los pros y contras en su ejecución, se han mantenido reuniones con los docentes para identificar las necesidades detectadas en la práctica tutorial. Asimismo, se ha trabajado con cuestionarios y entrevistas a estudiantes y profesores. Material que, tras ser analizado, ha generado un informe final que sirvió como base para el diseño del PAT.

Los alumnos encuestados fueron los de $1^{\circ}$ y $2^{\circ}$ de Grado -puesto que en el curso académico 2011-2012, en el que se ha realizado dicho trabajo, eran los cursos im- 
plantados en Grado en el Centro- y los de $5^{\circ}$ de Licenciatura -por entender que su mayor experiencia era imprescindible para el análisis-.

\section{Visión del alumnado y del profesorado del estado de la cuestión \\ 3.1. Valoración por el alumnado}

El uso de las tutorías es un procedimiento habitual para el alumno universitario. La mayoría del alumnado de Comunicación Audiovisual ha utilizado las tutorías que les ofrece el Centro. El 91,3\% de los alumnos las han utilizado alguna vez a lo largo de la carrera. Si bien, el porcentaje alcanza el $100 \%$ cuando se trata de los alumnos de $5^{\circ}$ de Licenciatura, el $98 \%$ en el caso de los de $2^{\circ}$ de Grado y se reduce al $76 \%$ en los de $1^{\circ}$.

En cuanto al número de veces que acuden a tutorías por materia y año, casi la mitad de los alumnos (49\%) utilizan las tutorías con una frecuencia que desde nuestro punto de vista consideramos como "imprescindibles" (de 1 a 3 por materia y curso). La frecuencia de 4 a 6 veces, que hemos valorado como tutorías "necesarias", se da en el $25 \%$ del alumnado. Sólo el $13 \%$ las utiliza en una medida que valoramos como tutorías "recomendadas" (de 7 a 9), y un 4\% en la que consideramos como "muchas" (10 o más). Opciones estas dos últimas sólo apuntadas por los alumnos de $2^{\circ}$ de Grado y $5^{\circ}$ de Licenciatura.

La mayoría de los alumnos considera las tutorías voluntarias bastante útiles. Así las han valorado el $43 \%$ de los encuestados, seguido de un $32 \%$ que las rebajan a medianamente útiles. Esta última opción es la más señalada por los alumnos de $1^{\circ}$ de Grado $(50 \%)$, frente a los de $2^{\circ}$ y $5^{\circ}$ de Licenciatura que las consideran bastante útiles ( $46 \%$ y $53 \%$ respectivamente). Ningún alumno cataloga las tutorías de nada útiles.

Las que se realizan con carácter obligatorio, un tipo de tutorías a las que han asistido el $67 \%$ de los alumnos encuestados, porcentaje que se eleva al $96 \%$ en los de $2^{\circ}$ de Grado -por la planificación obligatoria en el programa docente-, al igual que en el caso de las voluntarias, la mayoría de los alumnos, aunque en un porcentaje inferior, considera las obligatorias bastante útiles $(36,3 \%)$, prácticamente se mantienen los que las consideran muy útiles $(14,7 \%)$, pero aumenta el número de los que las valoran como poco útiles $(17,7 \%)$ e incluso aparece un porcentaje de alumnos que las considera nada útiles $(5,3 \%)$.

El momento más demandado para la realización de tutorías es un tiempo antes de la época de exámenes $(34,7 \%)$. La forma más utilizada de tutoría es la presencial dentro del horario de tutorías, señalada en un $77,7 \%$ de los casos. Asimismo, los alumnos optan también por el correo electrónico, en un $67,7 \%$ de los casos -se admitía más de una respuesta-. Los alumnos también han señalado como vía las tutorías presenciales pero fuera del horario de tutorías $(25,3 \%)$, poniendo de manifiesto la flexibilidad de los profesores, tan demandada por los alumnos.

La falta de tiempo es el principal motivo $(45,7 \%)$ por el que en alguna ocasión no han acudido a una tutoría a la que tenían pensado ir.

Es mayoritario el número de los que preferirían que las tutorías fueran concertadas, un 51,3\%. Cuando los alumnos han señalado como problemas de las tutorías los largos tiempos de espera porque se esté atendiendo a algún otro alumno o algún inconveniente relacionado con su propia gestión de los horarios, todos ellos señalan como sistema preferente las tutorías concertadas. 
Las principales razones señaladas por los alumnos para acudir a tutoría son la revisión de exámenes $(78,7 \%)$ y como guía del trabajo $(74,3 \%)$ y, aunque a distancia, para preguntar dudas del temario $(54,3 \%)$. Llama la atención que una cuestión como es la orientación profesional prácticamente es residual en los alumnos de $1^{\circ}(3 \%)$, adquiere algo más de importancia en los de $2^{\circ}(11 \%)$, pero es bastante importante en los de $5^{\circ}(43 \%)$. Los motivos personales son más frecuentes en los alumnos de $5^{\circ}(23 \%)$ que en los de $2^{\circ}(17 \%)$ y $1^{\circ}(3 \%)$, lo que puede tener que ver con su finalización de estudios o la mayor confianza con los profesores.

Finalmente, más de la mitad de los alumnos encuestados y de manera mayoritaria consideran que las tutorías podrían mejorarse con horarios más flexibles (58,3\%). Muy de lejos, los alumnos han hecho autocrítica y han señalado su mayor preparación en la materia a la hora de acudir a una tutoría como criterio de aumento de la calidad (26\%).

\subsection{Punto de vista del profesorado}

La visión del grupo de profesores de Comunicación Audiovisual con los que se ha trabajado ha coincidido con los datos aportados por los propios alumnos en cuanto a número de tutorías, momento de realización o forma más habitual, entre otras cuestiones.

Los docentes han entrado además a valorar la utilidad de las tutorías según el medio de realización, y consideran que las presenciales son las más útiles. En el caso de las realizadas a través del correo electrónico, la controversia surge por la dificultad de atender ciertas cuestiones a través de un correo, como la explicación y profundización en contenidos. Y además, porque a través de este medio se atienden muchas cuestiones del día a día del aula que el alumno debería conocer o simplemente las sabrían preguntando a sus compañeros.

Las tutorías no presenciales son atendidas de manera habitual, aunque muchos dicen que en ocasiones remiten a los alumnos a la presencial. Son muy pocos los que no las admiten. Las tutorías por e-mail o sistemas similares son generalmente contestadas en el mismo día, al día siguiente o en esa misma semana. La dedicación de tiempo a las mismas difiere de unos docentes a otros en función fundamentalmente del número de alumnos a su cargo y carga docente, dedicando, según el caso, entre 30 minutos hasta más de 2 horas semanales a la atención de correos. Por ello, considerarían oportuno la flexibilización del horario de tutoría presencial, dada la alta demanda y atención a las no presenciales.

Sobre las diferencias que pueden existir entre tutorías presenciales y no presenciales señalan una mayor utilidad de aquellas con respecto a éstas. Entienden que las presenciales permiten que se incorporen y resuelvan en su transcurso nuevas dudas, con una temática más amplia y con un trato más cercano. Las no presenciales tienen más que ver con faltas de asistencia o preguntas concretas sobre sus trabajos, siendo muy útiles a la hora de resolver dudas concretas, de manera rápida y eficiente. De forma mayoritaria se estaría dispuesto a utilizar herramientas como chat o videoconferencia para la realización de tutorías virtuales.

Son mayoría los profesores que prefieren que las tutorías se realicen con cita previa, pero son muchos los que las señalan sólo para algunos casos concretos, como la 
revisión de exámenes o trabajos. Es generalizado el que los alumnos en alguna ocasión hayan solicitado al profesor que les atiendan presencialmente con cita en algún momento fuera de su horario establecido de tutorías, por motivos laborales o personales. Solicitud que, igualmente, ha sido atendida de forma favorable por la práctica totalidad de docentes.

En general, los profesores consideran que el sistema de organización de tutorías del Centro es de calidad. La mayoría de estaría dispuesto a recibir formación en técnicas específicas para las tutorías, resultándoles además de utilidad una "guía de ayuda". Algunas cuestiones señaladas de forma específica para su posible mejora son la flexibilización y la planificación previa, que contribuye en gran medida a la mejora de las mismas, como se detecta perfectamente cuando son concertadas -un buen método de orientación y preparación de la tutoría a realizar-.

\section{Plan de Acción Tutorial: un sistema dinámico \\ 4.1. Planteamiento preliminar}

Partimos de la fijación de una definición de tutoría. Entendemos que es la acción de orientación presencial o virtual que el docente lleva a cabo sobre el alumno y que consiste en una ayuda particular entendida como algo intrínseco al proceso educativo del estudiante, que suscita su interés, estimula su comportamiento y propone metas significativas en su progreso académico. Teniendo en cuenta la diversidad de tutorías, nos centraremos en aquellas que, según su función, son consideras académicas.

Una vez conocido el estado de la cuestión en el CES Felipe II gracias a la combinación metodológica de lo cuantitativo y lo cualitativo, el objetivo general del PAT es coordinar todas las actividades de tutoría del centro universitario de tal forma que contribuya al completo desarrollo académico y curricular de los protagonistas de la acción didáctica.

Además, se establecen como objetivos específicos:

- Formar a personas íntegras en su conocimiento científico y en su comportamiento social.

- Estandarizar la relación tutorial con el alumno.

- Sistematizar y armonizar las acciones tutoriales para que el profesor reconozca su función orientadora y sepa atenerse a un protocolo de actuación concreto.

- Conseguir que los efectos beneficiosos del planteamiento lleguen a las esferas directivas.

- Lograr que el alumno tenga un manual de apoyo que le guíe en su relación tutorial con el docente.

- Mejorar la calidad del proceso de enseñanza y aprendizaje con el consiguiente aumento de la eficacia del sistema valorado con el parámetro de rendimiento académico.

En los siguientes cuadros se recogen el ámbito y las acciones del PAT y sus protagonistas y funciones, respectivamente. 
1. Ámbito y acciones del PAT. (Elaboración propia)

\begin{tabular}{|c|c|c|c|c|c|}
\hline Tipo & Acciones & & Modalidad & Meta & Funciones/ Observaciones \\
\hline \multirow{6}{*}{1} & \multirow{6}{*}{ Face to face } & \multirow{5}{*}{1} & \multirow{5}{*}{ Individuales } & \multirow{2}{*}{ Orientación personal } & Conocimiento del alumno \\
\hline & & & & & \multirow{2}{*}{$\begin{array}{l}\text { Orientación personal, } \\
\text { académica y profesional }\end{array}$} \\
\hline & & & & \multirow{2}{*}{ Orientación académica } & \\
\hline & & & & & Seguimiento personalizado \\
\hline & & & & Orientación profesional & Evaluación de resultados \\
\hline & & 2 & Grupales & \multicolumn{2}{|c|}{$\begin{array}{l}\text { El objetivo pasa por convertir el grupo en equipo } \\
\text { (cohesionado, con roles propios, con fines concretos) }\end{array}$} \\
\hline & & \multicolumn{4}{|c|}{ Potencia el uso y valor de esta actuación docente. } \\
\hline & & \multicolumn{4}{|c|}{$\begin{array}{l}\text { Aumenta el número de contactos, sin límite de tiempo, encontrando el } \\
\text { alumno una ventana abierta a su disposición en el momento del estudio o la } \\
\text { elaboración de trabajos que es cuando surgen las dudas. }\end{array}$} \\
\hline & & \multicolumn{4}{|c|}{ Agiliza y evita esperas, es rápida y asincrónica. } \\
\hline 2 & E-tutorías & \multicolumn{4}{|c|}{$\begin{array}{l}\text { Necesita de una actitud activa del alumno; y para el docente exige } \\
\text { formación, capacidad de adaptación, adecuación de sus habilidades de } \\
\text { comunicación a este registro, dominio de la técnica y sobre todo, un } \\
\text { volumen de tiempo que, muchas veces, el docente universitario no posee, es } \\
\text { decir, este recurso precisa de una exposición temporal que excede los } \\
\text { límites clásicos del horario -tradicional- de tutorías, siendo en ciertos } \\
\text { momentos abrumador el número de contactos solicitados por el alumnado a } \\
\text { través de las nuevas herramientas virtuales. }\end{array}$} \\
\hline
\end{tabular}

\section{Protagonistas y funciones del PAT (Elaboración propia)}

\begin{tabular}{|c|l|l|l|}
\hline $\begin{array}{c}\text { Tipología y } \\
\text { relación con } \\
\text { Universidad }\end{array}$ & \multicolumn{1}{|c|}{$\begin{array}{c}\text { Protagonistas del } \\
\text { PAT }\end{array}$} & \multicolumn{2}{|c|}{ Funciones y compromisos } \\
\hline 0 & Alumno (A) & 1 & $\begin{array}{l}\text { Asistir a las tutorías y responder al cuestionario } \\
\text { de calidad del PAT }\end{array}$ \\
\hline \multirow{2}{*}{1} & $\begin{array}{l}\text { Profesor de cada una } \\
\text { de las asignaturas } \\
\text { (PA) }\end{array}$ & 1 & $\begin{array}{l}\text { Fijar y cumplir las tutorías correspondientes a su } \\
\text { asignatura en el horario fijado }\end{array}$ \\
\hline \multirow{2}{*}{2} & \multirow{2}{*}{$\begin{array}{l}\text { Tutor (T) -por cada } \\
10 \text { alumnos- }\end{array}$} & 3 & $\begin{array}{l}\text { Dar a conocer los cambios en su acción tutorial } \\
\text { con un tiempo mínimo de dos días }\end{array}$ \\
\cline { 3 - 5 } & 2 & $\begin{array}{l}\text { Atender y orientar personal, académica y } \\
\text { profesionalmente al alumno }\end{array}$ \\
\cline { 3 - 5 } & & 4 & Eontribuir a la mejora del rendimiento \\
\cline { 3 - 5 } & & 5 & Astar en contacto con sus profesores \\
\cline { 3 - 5 } & 6 & $\begin{array}{l}\text { Preparar la documentación requerida sobre el } \\
\text { PAT }\end{array}$ \\
\hline
\end{tabular}




\begin{tabular}{|c|l|l|l|}
\hline 3 & $\begin{array}{l}\text { Coordinador de PAT } \\
\text { en cada Facultad (C) }\end{array}$ & 1 & $\begin{array}{l}\text { Asegurar la organización, coordinación y } \\
\text { seguimiento del Sistema de Tutorías de la } \\
\text { Facultad }\end{array}$ \\
\hline \multirow{2}{*}{4} & $\begin{array}{l}\text { Junta de } \\
\text { Coordinadores (JC) }\end{array}$ & 1 & $\begin{array}{l}\text { Vigilar el cumplimiento y desarrollo del PAT } \\
\text { en la Facultad }\end{array}$ \\
\cline { 2 - 4 } & 2 & Asesorar a los tutores en su labor \\
\hline \multirow{2}{*}{5} & $\begin{array}{l}\text { Responsable general } \\
\text { del PAT para toda la }\end{array}$ & 1 & $\begin{array}{l}\text { Asegurar la organización, coordinación y } \\
\text { seguimiento del Sistema de Tutorías de toda la } \\
\text { Universidad }\end{array}$ \\
\hline
\end{tabular}

\subsection{Fases y cronología del PAT}

En cuanto a la logística del Plan PAT, entendemos que para llevar a cabo la acción tutorial, se precisa que el profesor/tutor disponga de un tiempo en su jornada para atención de sus alumnos y entrevistas. Este tiempo será computado entre sus horas de dedicación docente (teniendo en cuenta que los componentes tradicionales de un Plan de Ordenación Docente son docencia, investigación y gestión). Hay que tener en cuenta que no sólo se considera tiempo de tutorías aquel que está el docente con el alumno, sino también el que utiliza el profesor para llevar a cabo el resto de las tareas relacionadas con el PAT (entrevistas, reuniones, redacción de informes y, sobre todo, atención a las e-tutorías, control y actualización de su Campus Virtual).

Asimismo, el Centro Universitario dispondrá de un lugar (físico u on-line) donde se anuncien los cambios en las tutorías de todos los profesores. Ese lugar tiene que recibir los cambios con una antelación mínima de dos días (salvo situación justificada).

Para evitar situaciones comprometidas al haber un cambio de ubicación momentánea del docente durante su horario de tutorías, se propone como requisito la cita previa, es decir, los alumnos escribirán al profesor/tutor un mail solicitándole la tutoría y dando una breve pincelada del tema a tratar. Esto facilitará que el profesor pueda preparar con antelación la acción tutorial y la atención y aprovechamiento del encuentro sea el más alto.

Cabe señalar además una serie de consideraciones:

- Este sistema se inspira en el proceso clásico de dirección de planificación, coordinación, motivación y control.

- Cada centro fijará el timing del PAT en función de su calendario académico, siendo consciente de que las reuniones iniciales tienen que desarrollarse en la primera semana de curso.

- La tipología de alumnos marcará, probablemente, un ajuste del PAT.

- Habrá que valorar y considerar como pertinente el conocimiento por parte del entorno primario y social de las líneas generales del PAT y, en caso del primero, situaciones excepcionales que pudieran acontecer.

Finalmente, la sistematización de nuestra propuesta del PAT resultaría como se recoge de forma sintetizada en el siguiente cuadro. 
3. Sistematización del PAT. (Elaboración propia)

\begin{tabular}{|c|c|c|c|c|}
\hline Orden & Fase & Responsable & & Tarea (por orden cronológico) \\
\hline \multirow{4}{*}{1} & \multirow{4}{*}{ Preliminar } & \multirow{4}{*}{ RG } & 1 & $\begin{array}{c}\text { Búsqueda de información y fijación } \\
\text { de la estructura }\end{array}$ \\
\hline & & & 2 & Planificación \\
\hline & & & 3 & Formación del tutor \\
\hline & & & 4 & Captación de recursos \\
\hline \multirow{12}{*}{2} & \multirow{12}{*}{ Desarrollo } & RG & 1 & Reunión inicial con la JC \\
\hline & & C & 2 & $\begin{array}{c}\text { Reunión inicial con cada uno de los } \\
\text { tutores } \\
\end{array}$ \\
\hline & & Cy RG & 3 & $\begin{array}{c}\text { Curso de orientación a los tutores (2 } \\
\text { horas máximo) }\end{array}$ \\
\hline & & PA & 4 & Fijar sus horas de tutorías \\
\hline & & $\mathrm{T}$ & 5 & Entrevista inicial \\
\hline & & A & 6 & Asistir a las tutorías \\
\hline & & PA & 7 & $\begin{array}{c}\text { Tutorizar a los alumnos en sus } \\
\text { asignaturas (face to face y e- } \\
\text { tutorías) }\end{array}$ \\
\hline & & $A, P A, T, C, R G$ & 8 & $\begin{array}{c}\text { Dar a conocer los cambios que } \\
\text { pudieran acontecer en todas las } \\
\text { fases }\end{array}$ \\
\hline & & $\mathrm{T}$ & 9 & $\begin{array}{c}\text { Entrevista periódica, informativa y } \\
\text { de diagnóstico }\end{array}$ \\
\hline & & $\mathrm{T}$ & 10 & $\begin{array}{c}\text { Entrevista de seguimiento y } \\
\text { evaluación }\end{array}$ \\
\hline & & $\mathrm{T}$ & 11 & Entrevistas ocasionales \\
\hline & & $\mathrm{T}$ & 12 & Comunicación formal e informal \\
\hline \multirow{4}{*}{3} & \multirow{4}{*}{ Evaluación } & $\mathrm{T}, \mathrm{C}, \mathrm{JC}, \mathrm{RG}$ & 1 & Análisis \\
\hline & & $A, T, C, R G$ & 2 & Evaluación \\
\hline & & $P A, T, C, R G$ & 3 & $\begin{array}{l}\text { Redacción de informes, memoria } \\
\text { final y propuestas de mejora }\end{array}$ \\
\hline & & RG & 4 & Evaluación global sobre el PAT \\
\hline
\end{tabular}

\section{Conclusiones}

Diversas universidades han superado ya la primera etapa de aplicación del EEES y se enfrentan ahora al análisis de la repercusión del nuevo planteamiento en la labor docente, otras están a las puertas de la implantación. En todo caso, este paso se está dando en diferentes facultades de Comunicación que, intentando colocarse a la vanguardia de los cambios, estudian cuál ha sido el reflejo de las necesarias transformaciones en la docencia de nuestras ciencias, un campo que desde hace tiempo y en diversos foros, demanda (debido, tal vez, a su currículum epistemológico necesariamente joven) una conceptualización vanguardista y una práctica pegada a una realidad laboral cambiante.

En esta línea, las diferentes asignaturas se esfuerzan por progresar e implantar nuevas estrategias y metodologías de enseñanza-aprendizaje, entre ellas, las vinculadas a 
las tutorías. Ahora sólo falta que se cumpla lo que dice Ferrer (2003) al afirmar que un Plan de Acción tiene entre sus objetivos estimular la reflexión colectiva acerca de qué, cómo, para qué y a quién se enseña; difundir entre los profesores información precisa sobre objetivos, organización docente, itinerarios curriculares, contenidos teóricos y prácticos y métodos de evaluación para su análisis y posterior reforma; establecer unas formas de relación entre profesor y alumno que vayan mucho más allá de las que se dan en las aulas o a través de las consultas concretas de carácter instrumental; aproximar los métodos de trabajo de la universidad a los que son propios en el ejercicio de las profesiones; caracterizar al estudiante como protagonista de su proceso de formación; estimular procesos de autoevaluación de los profesores a partir de la información que suministran los tutelados; mejorar el proceso de inserción laboral de los titulados; mejorar la calidad del proceso de enseñanza y aprendizaje con el consiguiente aumento de la eficacia del sistema valorado con el parámetro de rendimiento académico.

Quizás sean demasiados fines. Pero hay que tener en cuenta que con esfuerzo y miras en lo excelso, se llega a cumplir con lo conveniente. Hay está nuestra meta.

\section{Referencias bibliográficas}

ALCOLEA, Gema y PÉREZ SERRANO, María José (2010): "Las e-tutorías en dos áreas troncales de comunicación adaptadas a Bolonia. Ventajas y dificultades", en SIERRA SÁNCHEZ, Javier y SOTELO GONZÁLEZ, Joaquín (coords.): Métodos de innovación docente aplicados a los estudios de Ciencias de la Comunicación. Madrid, Fragua.

DÍAZ-GUILERA, Albert (2004): "Los planes de acción tutorial en la Universidad de Barcelona", en Programa de formación y atención al profesor tutor. Barcelona, Universitat de Barcelona. Disponible en http://www.upcomillas.es/eees/Documentos/acciontutorialubarcelona.pdf Consultado el 7 de mayo de 2011.

FERRER, Josep (2003): "La acción tutorial en la universidad", en MICHAVILA PITARCH, Francisco F.; GARCÍA DELGADO, Javier (coords.): La tutoría y los nuevos modos de aprendizaje en la universidad. Madrid, Consejería de Educación.

GARCÍA RAMOS, José Manuel y GÁLVEZ HERNÁNDEZ, María (1996): “Un modelo tutorial universitario". Revista Complutense de Educación, vol. 7, n 1 . Madrid, Servicio de Publicaciones Universidad Complutense.

MARTÍNEZ RODRÍGUEZ, Azucena y MARTÍNEZ RODRÍGUEZ, Orquídea (2011): "Identificación de necesidades para el diseño de un modelo de tutorías en la educación superior". Tlatemoani. Revista Académica de Investigación, núm. 5, marzo 2011. Disponible en http://www.eumed.net/rev/tlatemoani/ $/ 05 / \mathrm{mrmr}$.htm Consultado el 8 de agosto de 2011.

MIRAFLORES GÓMEZ, Emilio (2004): "La acción tutorial en centros universitarios de Magisterio de la Comunidad de Madrid. Propuesta de futuro". La nueva alfabetización: un reto para la educación del siglo XXI, CES Don Bosco. Madrid. Disponible en http://www.cesdonbosco.com/revista/congreso/37-Emilio\%20Miraflores\%20Gomez.pdf Consultado el 9 de julio de 2011. 
RODRÍGUEZ URÍA, María Victoria y otros (2007): "La acción tutorial en la Universidad en el marco del Espacio Europeo de Educación Superior". XV Jornadas de ASEPUMA y II Encuentro Internacional. Disponible en http://www.uv.es/asepuma/XV/comunica/606.pdf Consultado el 9 de septiembre de 2011.

\section{Gema ALCOLEA DÍAZ}

gema.alcolea@ajz.ucm.es

CES Felipe II, Universidad Complutense de Madrid

\section{María José PÉREZ SERRANO}

mariajoseperezserrano@pdi.ucm.es

Universidad Complutense de Madrid 